\title{
Legal modeling as method for studying genetic and genomic information
}

\author{
Darina Sergeevna Vladimirova* \\ Ural State Law University, Ekaterinburg, Russia
}

\begin{abstract}
The relevance of this work is due to the fact that genomic and genetic information are special objects of research for law, and they require a systematic approach able to regard as many features of such information as possible. It seems that legal modeling is one of the key legal research methods capable of coping with this task. The purpose of this study is to consider the method of legal modeling in the study of genetic and genomic information. The paper outlines some aspects of its application, its advantages for interdisciplinary research. It has been established that legal modeling is able to regard all the factors influencing the legal regulation of genetic and genomic information. The staging of legal modeling, which implies determining the object and the subject of legal modeling, setting goals and objectives, proposing and testing a hypothesis and formulating a legal model, has been noted.
\end{abstract}

\section{Introduction}

Theoretical and legal research becomes relevant for systemic and complex social phenomena that go far beyond the boundaries of law. Genetic research and genetic information are not only bound to a whole range of natural sciences but also are the subject of study in many humanities since they affect universal human values, knowledge of a person as a product of spiritual activity, his fundamental rights and freedoms. Currently, there is a significant increase in conducing legal research on genetics. Such legal research does not relate to any branch of law, their specificity presupposes an integrated systematic approach to solving emerging issues, which has actualized the interdisciplinary research.

Currently, the legal regulation of genomic research is impeded by the complexity of this concept, which requires a systematic approach. The available research, as a rule, considers certain aspects, which leads to a multitude of viewpoints in the absence of common concepts and provisions; as well, there is lack of such unity in a situation when the term "genome" is used [1].

The purpose of legal regulation is to prevent the misuse of genomic information. The problem is aggravated by the fact that at the present stage, the consequences of using such information and its potential are not entirely clear. Therefore, some of the readily available genetic research even now may pose a very real threat to society. The problem of legal regulation in this regard is both the lack of certainty in perceiving the term "genetic information", and the procedure for conducting genetic testing and using, storing its results [2]. Thus, the increased relevance of legal work in the field of genetics is still urgent. However, there is a methodological problem in research related to the specificity of genomic relationships.

The necessary techniques and methods of cognition increase the quality of any research and benefit a rational distribution of research potential, which is effective in scientific research. A correctly chosen method contributes to getting true knowledge objectively reflecting the reality.

Taking into account the consistency of genomic relations, it seems relevant to use legal modeling as a research method assisting in identifying the general trends inherent in the entire complex of genomic legal relations.

This present article aims to consider the possibility of using legal modeling in the study of social relations arising from genomic and genetic information, to develop methodological issues comprehension in the complex and multifaceted area of genomic legal relations.

\section{$2 \quad$ Materials and methods}

The methodological basis of the research is a combination of both general scientific and special legal methods. The general scientific methods include: analysis, synthesis, induction, deduction. Granting the designated research problems, the work mainly used a systemic and integrative approach, as well as a formal legal method.

\section{$3 \quad$ Results and Discussion}

Legal modeling is currently not a common method used in humanitarian research but it appears that it can solve 
some of the issues arising in the study of genomic and genetic information.

Law is a systemic education that affects almost all areas of society. Thus, the object of study poses the need to use a holistic approach and methodological diversity in jurisprudence [3]. Interdisciplinary research requires a systematic approach, which in turn leads to the need to create complex phenomena for the most effective study of a little-known category of social relations.

Legal regulation does not comply with experiments. Therefore, the introduction of new norms of law being verified and consistent with the principles and values of law is a very long and complex process. The specificity of social relations that develop in connection with genomic information, their significant social and economic significance, deep connection with ethics and natural human rights do not allow establishing an unambiguous legal regulation without a thorough consideration of all emerging problems and controversial situations.

It seems that it is legal modeling that is capable of creating some universal tools for research and obtaining relevant results in this area. In order to cover such complex social relations "it is necessary to use a universal legal category that would allow studying such a complex subject of research" [4].

In this case, legal modeling can solve some problems since it is possible to build abstract legal models, check their effectiveness without harming one or another interests of society and a particular citizen.

The specificity of modeling as a method of legal research, which has not previously been used as such in humanitarian works, is widely noted. The success of using this method in the natural and technical sciences could not have been ignored, and over time, this method was consistently introduced and adapted, which was initially unusual for it.

In the literature, modeling is understood as a research method in which "the object of interest to the researcher is replaced by another object similar to the first object. The first object is called the original and the second is called the model. In the future, the knowledge gained during the model studying is transferred to the original base on analogy and the theory of similarity" [5].

In another source, modeling is understood as "a method of indirect practical or theoretical object manipulation, when not the object of interest is directly investigated but an auxiliary artificial or natural system ("quasi-object") is used, which is in a certain objective correspondence with the object being cognized, capable of replacing it at certain stages of cognition and ultimately giving information about the modeled object itself during its research [6].

Modeling is relevant when studying homogeneous social relations [7]. "Modeling as a method of studying legal (and generally social) phenomena enables to regard a concept being the unity, sameness of relations described by a theoretical construction" [8].

The method of legal modeling refers to the special technical and legal method. The model is the main design tool. Real social relations arising from genetic and genomic information are complex and multifaceted.
The modeling method can serve as the most optimal tool for the cognition of such relations and the most effective method of their study.

A.F. Cherdantsev noted that the model may not have a research nature but perform a special task of description and demonstration since the study of legal phenomena is not always a goal in itself [9]. The existing research is miscellaneous and fragmented. Thus, modeling can create a complete picture of existing research. Consequently, legal modeling performs an important epistemological function in the study of genetic and genomic information.

One of the aspects of applying the method of legal modeling can be the creation of model legislation and norms, which together with a legislative experiment can help in solving many issues arising in the field of legal regulation of genetics. At the moment, there is no relevant model legislation even at the level of international organizations, although the relevant norms can be developed with regards to the existing conventions and positions of international judicial bodies.

The main intended purpose of legal modeling is the ability to predict the development of legal regulation and its results. In genetics development, lawyers are faced with the task of simulating the process of this regulatory legal act operation, its integration into the system of legal, social, economic and political values, possible scenarios for the subsequent development of social relations.

In legal modeling, attention should be paid precisely to behavior, which, as a result of legal impact, can both comply with the norms of law and go beyond the framework of its regulation. This understanding enables to create practical predictive models.

Genomic information should be legally regulated through the establishment of both regulatory and protective norms. In domestic legislation, there is actually only one normative legal act which contains special provisions, specifically, the Federal Law "On State Genomic Registration in the Russian Federation". The rest is regulated by laws that regulate genomic and genetic information precisely because of its multifaceted nature: medical secrecy, personal information, etc.

An example of modeling in relation to genomic relationships is the legislative definitions that exist today. At the moment, Russian legislation has only one definition related to genomic information, which is contained in Article 1 of the Federal Law "On State Genomic Registration". Eventually this definition becomes irrelevant for many areas of legal regulation, since it contains only one defining legal feature that classifies such information as personal. Thus, there is a need to create universal terms and their definitions.

The meaning of legislative terms and definitions in the field of genomic relations, of course, cannot be idealized due to the complex structure of research objects and the variety of social relations in which they can appear. However, it is possible to choose an aspect that would take into account the legal interest and solve the problems arising when applying and implementing the law. Since today the integration of the principles of 
bioethics into the legal field is of particular relevance in the field of genetic and genomic information, it is possible to formulate legislative definitions in the context of fundamental human rights and freedoms. This will allow gradually expanding the legal potential and other model norms in the field of science, health care and human well-being.

The relationship with regard to genomic and genetic information is complex. Legal modeling can be an effective way to study legal relations arising in connection with genetic and genomic research since this method systematizes and organizes legal relations regarding genomic information.

In the traditional sense, modeling is a cognitive method that consists in creating and researching models. A model is a kind of new object that reflects the essential features of the studied object, phenomenon or process.

Since a considerable number of issues in the field of genetic information have matured with regards to the theoretical and legal field, the solution of these issues is seen in the creation and involvement of verified wellconsidered theoretical models and mechanisms for their implementation into practical legal activity. As noted in the studies, "a properly thought-out legal model contributes to both introduction of the object of the study itself and its subsequent use for reforming other related legal relations based on the conclusions it received" [10].

The modeling method is distinguished by the reference to a systematic approach to the study of legal reality, which implies the use of a set of humanitarian knowledge and not only when creating a model. This is relevant for genetic relations since some questions arising in the study of such a complex object belong to ethics, philosophy, sociology, political science and other fields. In this connection, the difficulties in creating an appropriate legal model may be the need to take into account all the components and directions of the system.

The practical value of legal modeling both in the study of genomic and other social relations lies in the fact that it becomes possible to predict the events development, assess the effectiveness or ineffectiveness of one or another state-legal decision.

At the same time, it is necessary to consider the disadvantages of using legal modeling in research activities. Since modeling is a method that is still characteristic of the natural and exact sciences, where it is more likely to investigate and predict reality, the specificity of the humanities presupposes the absence of such accuracy and the inability to predict the consequences and development of situations with sufficient accuracy. Legal modeling with regard to genomic relations can only predict certain options for the development of a particular topic with a sufficient degree of probability since such social ties are subject to a significant influence of external and internal factors, which are also not in the reach of the subject of cognition.

A feature of applying legal modeling in the study of genomic and genetic information is the possibility of constructing theoretical models that may contain knowledge about the structure and functions of the system and are relevant for being used in different contexts [11].

Legal modeling is essential for the development of genetics. The following modeling functions can be distinguished, which will most clearly reveal the value of using such a method in such specific social relations: 1) the epistemological function, in accordance with which the process of cognizing reality, the study and demonstration of the acquired knowledge takes place; 2) a prognostic function, thanks to which it is possible to work on the ideas of the social connections development regarding genetic and genomic information.

It seems that it is the prognostic function that should be given more attention at the moment. Since the field of genetics is currently a new field, and the knowledge gained in this area has enormous potential, whose fulfillment is possible in compliance with both negative and positive scenarios. The creation of forecasting models will be able to most effectively solve the assigned tasks, minimize risks and avoid certain negative scenarios that are very destructive for this sphere of public relations.

The whole spectrum of genomic relations like any other social relations is at the mercy of regulating various social norms but not only that. Factors that do not fall within the spectrum of rationing have a significant impact on genomic relations [12]. Since genomic information is of great social importance and affects areas that are highly sensitive and problematic for human consciousness, the main task is to maximally develop a legal regulation able to regard all controversial issues [13].

The main result of modeling is the construction of a model, which is a mental or material reflection of real phenomena. Modeling is applicable to complex, systemic objects, the real study of which is difficult. Modeling, on the other hand, simplifies the real object by giving meaning only to those properties of the object that are of cognitive interest.

In the methodological literature on legal modeling, there are two requirements for creating a model. Specifically, they are adequacy to the original and universality.

The object of legal modeling will be public relations arising from genomic and genetic information. The subject of legal modeling is already specific social relations, which should be included in the subject of legal regulation or in respect of which the mode of appropriate regulation is being revised leading to appropriate legal ties establishment.

The legal modeling process can consist of several stages. At the preparatory stage, the actors involved in the creation of the corresponding models should be determined. The specificity of genetic relations implies the presence of certain knowledge not only in the field of law, philosophy, religious studies, sociology but also in the field of natural sciences, since only awareness of the specifics of genetics and its potential will enable to create a more complete model.

At the second stage, the problem of legal modeling is posed. As noted above, genetic relationships are complex structural relationships involved in many 
different institutional relationships. Thus, the goals and objectives of legal modeling are formulated.

At the third stage, a direct meaningful legal model is created in the field of genetic or genomic information. The model includes all the essential properties of the object and subject of the relevant social relations in the field of genetics, which are necessary and sufficient for the tasks as well as for the prognostic purposes. At this stage, hypotheses are put forward based on the already known data. It should be noted that some social relations in the field of genetics have general regulation. Thus, it is possible to create legal models based on the existing regulation with a greater degree of reliability.

Within the framework of legal modeling, a model for the protection of individual rights can be created today, allowing to take into account private and public interests in the process of obtaining, storing, using genomic and genetic information. Such a model can be a combination of methods of protection, various measures of the state and, possibly, public response, a system of special competent authorities, endowed with appropriate powers.

\section{Conclusion}

Summarizing the above, the following conclusions can be drawn:

1. The social relations developing with regard to genomic and genetic information are complex structural relations, whose specificity must be reflected both in theoretical research in the field of law and in the legal regulation itself.

2. Legal modeling can be a suitable method for studying such relations, which is of great practical importance while it enables to regard many factors influencing the formation of genomic legal relations, and to predict the development of legal ties and their impact on all spheres of public life.

3. Legal modeling performs rather essential epistemological and prognostic functions in the study of genetic and genomic information. The process of legal modeling consists of several stages and requires defining the object and subject of modeling, setting goals and objectives, and advancing the working hypotheses in relation to genetic and genomic information based on all existing institutional ties. The validity of such hypotheses depends on the novelty of legal prescriptions and consideration of all factors that may affect the development of social relations.

4. With a view to further development of the field related to genetic and genomic information, with the appropriate effective development of legal regulation in this matter, the priority seems to be the development of model normative legal acts and the creation of an effective model for the protection of individual rights.

The research was funded by subsidies from the federal budget for a scientific project "Identification of legal regulatory problems in the use of genetic and genomic information. Preparation of scientifically sound proposals to amend the system of Russian legislation in order to eliminate them" (subject code: FEUW-2020-0005)

\section{References}

1. L. N. Berg, V. G. Golubtsov, Areas of Legal Impact in the Field of Genomic Research: Russian and International Experience, Perm University Herald. Juridical Sciences, 50, 641 (2020). DOI: 10.17072/1995-4190-2020-50-638-649.

2. A. Bevan, D. Saragoussi, L. Sayegh, M. Ringo, F. Kearney, Genetic Testing in Natural History Studies: A Review of the Regulatory and Legal Landscape, Public Health Genomics, 10-12 (2021). doi: 10.1159/000514208.

3. B. A. Kistyakovsky, Social Sciences and Law: Essays on the Methodology of the Social Sciences and General Theory of Law (M. and S., Moscow, 1916) $718 \mathrm{p}$.

4. L.N. Berg (ed.), Genome, man, law: problems of theory and practice of legal impact (Jurlitinform, Moscow, 2021) $200 \mathrm{p}$.

5. P.V. Alekseev, A.V. Panin, Philosophy: Textbook (Moscow, 2005) p. 376.

6. I.B. Novik, On the modeling of complex systems (philosophical essay). (Mysl', Moscow, 1965) 335 p.

7. K.V. Shundikov, Synergetic Approach in Legal Science: Problems of Adaptation, 1(276), 145-156 (2008)

8. V.D. Rudashevsky, Law and modeling. In: Methodological problems of the Soviet legal science (Science, Moscow, 1980) p. 303.

9. A.F. Cherdantsev, Interpretation of law and contract: Tutorial (Unity-Dana, Moscow, 2003) 382 p.

10. I.V. Fatyanov, State-legal modeling: concept and place in the system of methods of the theory of state and law, Bulletin of Tomsk State University, 416, 191-195 (2017)

11. S. Slokenberga, You can't put the genie back in the bottle: On the legal and conceptual understanding of genetic privacy in the era of personal data protection in Europe, University of Trento, S1, 231 (2021). DOI: $10.15168 / 2284-4503-783$.

12. C. Schickhardt, H. Fleischer, E.C. Winkler, Do patients and research subjects have a right to receive their genomic raw data? An ethical and legal analysis, BMC Med Ethics, 21(7), 4 (2020)

13. T. Faunce, A. Ray, C. Gardiner, T. Preiss, G. Burgio, Regulating RNA Research and CRISPR Gene Drives to Combat Biosecurity Threats, J. Law Med, 26(1), 212 (2018) 